\title{
Modeling and Motion Analysis of the MARES Autonomous Underwater Vehicle
}

\author{
Bruno Ferreira, Miguel Pinto, Aníbal Matos, Nuno Cruz \\ FEUP - DEEC \\ Rua Dr. Roberto Frias, s/n 4200-465 Porto PORTUGAL \\ ee04018@fe.up.pt, ee04134@fe.up.pt, anibal@fe.up.pt, nacruz@fe.up.pt
}

\begin{abstract}
In the robotic domain, it is common to deduce and use models that allow translating mathematically the element behavior. In some cases, these would serve as base to determine and develop a controller, for example. Beyond this, the simulation and experiments are reasons that leave to the development of models, becoming evaluation tools of the system behavior, especially when there are constraints of implementation or in experiments. However, the modeling is an approach to the reality, since it is difficult to translate the behavior of an element in a strict way and the disturbances to witch it is subject to. In this work, we address the modeling questions of an autonomous underwater vehicle.

This paper describes the deducing of a dynamic model with six degrees of freedom of an underwater vehicle, considering all of its physical characteristics. This is achieved by the determination of all forces that actuates on the body during its motions and by the determination of the rigid body dynamic. The modeling method is presented as well as the coefficients determination. Finally, a comparison with experimental results is carried out.
\end{abstract}

\section{INTRODUCTION}

Underwater robotic is often affected by experimental constraints. In most of cases, first tests of new implementations must be done in closed environment as a pool or a tank, preventing any error. This is especially true when the autonomy of the robot increases.

The particular case of MARES [1-2] (fig. 1-3) needs large space to move during tasks of its major application areas. Most of missions that it performs imply diving to several tens of meters as motion in a limited horizontal plane with some hundreds of meter wide. Such dimensions are only available in a river, lake or ocean. These environments represent risks for tests, in case of failure.

In this context, the determination of a model may be useful, taking into consideration that the behavior of the vehicle may be predicted. Beyond this, the implementation of a simulator based on the model allows knowing of followed trajectories. If, in addition, constraints due to the environment are modeled, a good approach to the real behavior may be obtained.

In other hand, a model may be used to determine control laws that allow performing of several trajectories as it is demonstrated in [3-5].

It is important that the model closely characterize the dynamic of the vehicle motion. To reach this aim, all major characteristics must be considered.

\section{MARES AUV}

MARES, or Modular Autonomous Robot for Environment Sampling, is a $1.5 \mathrm{~m}$ long AUV (Autonomous Underwater Vehicle), designed and built by the Ocean Systems Group at the Faculty of Engineering of University of Porto. The vehicle can be programmed to follow predefined trajectories, while collecting relevant data with the onboard sensors. MARES has a slender body form and is endowed with four thrusters that confer it four controllable degrees of freedom (DOF). It can dive up to $100 \mathrm{~m}$ deep, and unlike similar-sized systems, has vertical thrusters to allow a purely vertical motion in the water column. Forward velocity may be independently defined, from 0 to about $1.5 \mathrm{~m} / \mathrm{s}$. Major application areas include pollution monitoring, scientific data collection, sonar mapping, underwater video or mine countermeasures.

Though MARES may have multiple configurations, we will assume only one as shown in fig.1-3.

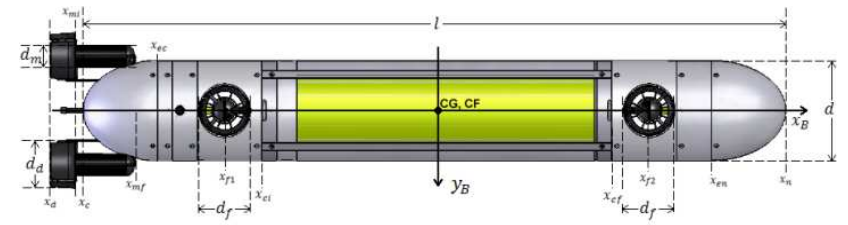

Fig. 1: Superior view of MARES.

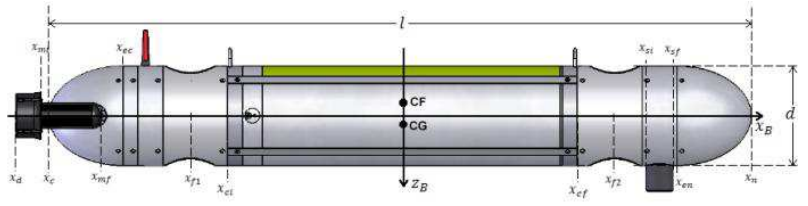

Fig. 2: Lateral view of MARES

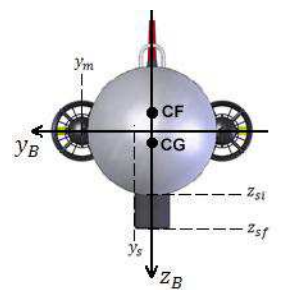

Fig. 3: Frontal view of MARES

As we can see, the vehicle is symmetric relatively to the plane formed by $x_{B}$ and $y_{B}$ axis. This characteristic will be helpful on simplification of forces modeling. Additionally, it is empirical that the motion in the $z_{B}$ and in the $y_{B}$ directions will originate different forces, due to hull orifices. In the first case the water pass through orifices but not in the second. This 
behavior will be explored in the modeling section. The effects due to the antenna and handles, on the superior part of the vehicle, will be neglected.

\section{MODELING OF THE DYNAMIC}

In this section, we present a general method to determine a model for a body inserted in a fluid. We start by relating the sum of forces that actuate in the vehicle with its acceleration through the Newton second law, prosecuting with the forces identification.

\section{A. Kinematic}

In order to simplify the deducing of model expressions, we consider an earth-fixed and a body-fixed referential [6]. Both are orthogonal. We assume that the earth-fixed referential, formed by axes set $\{x, y, z\}$, do not experiences any acceleration, i.e., it is inertial. Body-fixed referential is formed by $\left\{x_{B}, y_{B}, z_{B}\right\}$ as it is shown in figures 1-3.

A linear vector $c_{B} \in \mathbb{R}^{3}$ in the body-fixed referential may be expressed in the earth-fixed referential through the relation

$$
c=J \cdot c_{B},
$$

where $J \in \mathbb{R}^{3 \times 3}$ is the rotation matrix of the referential $\{x, y, z\}$ from $\left\{x_{B}, y_{B}, z_{B}\right\}$. Note that $J$ is orthonormal and its inverse is given by $J^{-1}=J^{T}$.

\section{B. Rigid-body dynamic}

The Newton's second law states that a rigid body at rest only experiences motion if a force is applied to it. In the inverse case, a body only reaches the rest state if a force counters the motion. Mathematically, these concepts are translated by following two expressions that represent conservation of linear and angular momentum, respectively:

$$
\begin{gathered}
\int_{V} \frac{d^{2} r}{d t^{2}} \rho_{B}\left(r_{G}\right) d V=\int_{V} \rho_{B}\left(r_{G}\right) g d V+\int_{S} f\left(r_{G}\right) d S, \\
\int_{V} \frac{d}{d t}\left(r \times \frac{d r}{d t}\right) \rho_{B}\left(r_{G}\right) d V=\int_{V} r \times \rho_{B}\left(r_{G}\right) g d V \\
+\int_{S} r \times f\left(r_{G}\right) d S,
\end{gathered}
$$

where $r$ and $r_{G}$ are the position of a point of the body related to the earth-fixed inertial referential and related to the bodyfixed referential, respectively, $V$ is the volume of the body, $\rho_{B}$ the density, $S$ the surface, $g$ the acceleration of the gravity and $\cdot x \cdot$ represents the cross product.

The development of these two equations gives the sum of exterior forces and moment that actuate on the body, respectively:

$$
\begin{gathered}
\sum f_{\text {ext }}=m\left(\dot{v}_{r}+\omega_{B} \times v_{B}+\dot{\omega}_{B} \times r_{G}+\omega_{B}\right. \\
\left.\times\left(\omega_{B} \times r_{G}\right)\right) \\
\sum m_{e x t}=m r_{G} \times \dot{v}_{r}+m r_{G} \times\left(\omega_{B} \times v_{B}\right)+I_{B} \dot{\omega}_{B} \\
+\omega_{B} \times\left(I_{B} \omega_{B}\right)
\end{gathered}
$$

where $\sum f_{\text {ext }}=\left(\sum X_{\text {ext }}, \sum Y_{\text {ext }}, \sum Z_{\text {ext }}\right)^{T}$ is the vector of forces components in $x_{B}, y_{B}$ and $z_{B}$ axes, $\sum m_{\text {ext }}=$ $\left(\sum K_{\text {ext }}, \sum M_{\text {ext }}, \sum N_{\text {ext }}\right)^{T}$ is the vector of moments components after $x_{B}, y_{B}$ and $z_{B}$ axes, $m$ is the mass of the vehicle, $I_{B}$ is the inertia tensor with respect to the body-fixed origin, $\dot{v}_{r}$ is the time derivative of the velocity in the bodyfixed referential, $v_{B}$ and $\omega_{B}$ are linear and angular velocities of the body in the earth-fixed referential, respectively, and $\dot{v}_{B}$ and $\dot{\omega}_{B}$ their derivative in order of time.

The deducing of (4) and (5) may be consulted in [7]. Designating $\tau_{\text {ext }}=\left[\sum f_{\text {ext }} \sum m_{\text {ext }}\right]^{T}$ as the vector of forces and moments, we may write

$$
\tau_{\text {ext }}=M_{R B} \dot{v}+C_{R B}(v) v,
$$

where $M_{R B}, C_{R B}(v) \in \mathbb{R}^{6 \times 6}$ are the rigid body inertia matrix and the Coriolis and centrifugal terms matrix, respectively. These matrices are given by

$$
M_{R B}=\operatorname{diag}\left(m, m, m, I_{x x}, I_{y y}, I_{z z}\right),
$$

where $I_{i i}$ is the moment of inertia after the $i$-axis, and

$$
C_{R B}=\left[\begin{array}{cccccc}
0 & -m r & m q & 0 & 0 & 0 \\
m r & 0 & -m p & 0 & 0 & 0 \\
-m q & m p & 0 & 0 & 0 & 0 \\
0 & 0 & 0 & 0 & I_{z z} r & -I_{y y} q \\
0 & 0 & 0 & -I_{z z} r & 0 & I_{x x} p \\
0 & 0 & 0 & I_{y y} q & -I_{x x} p & 0
\end{array}\right],
$$

considering that the body-fixed referential coincides with the center of gravity of the body and that products of inertia ( $I_{i j}$ for $i \neq j$ ) are negligible.

\section{Hydrodynamic}

A body inserted in a fluid experiences several forces. In the particular case of underwater vehicles, these forces are due to:

- Added mass, originated by the acceleration of involving particles of fluid during the acceleration of the body;

- Drag, due to friction on the boundary layers, pressure on the hull and vortices created by non null velocity;

- Potential damping that is originated by non null velocity of the body. Its contribution is small compared to drag and is often included on it. In this paper, we will do the same;

- Froude-Krylov force due to the acceleration of the fluid;

- Restoring forces due to the weight and to the buoyancy;

- Propulsion exercised by actuators.

Note that forces induced by the wind and by waves are neglected, assuming that, for underwater vehicles performing motion sufficiently far from the surface, these effect are relatively small. We recall that the antenna and handles are ignored, considering their small dimensions.

\section{Added mass}

The symmetry of the MARES AUV after the plane formed by $\left\{x_{B}, z_{B}\right\}$ axes allows the simplification of the added mass force expression that is given by

$$
\tau_{A}(\dot{v}, v)=-M_{A} \dot{v}_{r}-C_{A}\left(v_{r}\right) v_{r},
$$


where $M_{A}, C_{A}(v) \in \mathbb{R}^{6 \times 6}$ are the added mass matrix and Coriolis and centrifugal terms matrix, respectively.

In order to clarify the simplification method we consider following examples:

- Linear acceleration after the $x_{B}$ axis does not generate any moment after $z_{B}$, which implies that the corresponding term in $M_{A}$ must be null $\left(N_{\dot{u}}=0\right)$;

- Angular acceleration after the $y_{B}$ axis will not have any influence on the force after the same axis;

- Linear acceleration after $z_{B}$ does not create any force after $x_{B}$ or $y_{B}$ axis.

Applying the same reasoning to others terms, we obtain

$$
M_{A}=-\left[\begin{array}{cccccc}
X_{\dot{u}} & 0 & 0 & 0 & X_{\dot{q}} & 0 \\
0 & Y_{\dot{v}} & 0 & Y_{\dot{p}} & 0 & Y_{\dot{r}} \\
0 & 0 & Z_{\dot{w}} & 0 & Z_{\dot{q}} & 0 \\
0 & K_{\dot{v}} & 0 & K_{\dot{p}} & 0 & 0 \\
M_{\dot{u}} & 0 & M_{\dot{w}} & 0 & M_{\dot{q}} & 0 \\
0 & N_{\dot{v}} & 0 & 0 & 0 & N_{\dot{r}}
\end{array}\right]
$$

and

$$
C_{A}(v)=\left[\begin{array}{cccccc}
0 & 0 & 0 & 0 & C_{A}^{15} & C_{A}^{16} \\
0 & 0 & 0 & -C_{A}^{15} & 0 & C_{A}^{26} \\
0 & 0 & 0 & -C_{A}^{16} & -C_{A}^{26} & 0 \\
0 & C_{A}^{15} & C_{A}^{16} & 0 & C_{A}^{45} & C_{A}^{46} \\
-C_{A}^{15} & 0 & C_{A}^{26} & -C_{A}^{45} & 0 & C_{A}^{56} \\
-C_{A}^{16} & -C_{A}^{26} & 0 & -C_{A}^{46} & -C_{A}^{56} & 0
\end{array}\right],
$$

where

$$
\begin{gathered}
C_{A}^{15}=-Z_{\dot{w}} w-Z_{\dot{q}} q \\
C_{A}^{16}=Y_{\dot{v}} v+Y_{\dot{p}} p+Y_{\dot{r}} r \\
C_{A}^{26}=-X_{\dot{u}} u-X_{\dot{q}} q \\
C_{A}^{45}=-Y_{\dot{r}} v-N_{\dot{r}} r \\
C_{A}^{46}=X_{\dot{q}} u+Z_{\dot{q}} w+M_{\dot{q}} q \\
C_{A}^{56}=-Y_{\dot{p}} v-K_{\dot{p}} p
\end{gathered}
$$

It is important to refer that coefficients like $Y_{\dot{r}}, Z_{\dot{q}}, M_{\dot{w}}$ and $N_{\dot{v}}$ are non null due to the asymmetry relatively to $\left\{y_{B}, z_{B}\right\}$ plane. The sonar hull, on the inferior part of MARES (cf. fig. $2-3)$, turns the body asymmetric after the $\left\{x_{B}, y_{B}\right\}$ plane, making coefficients $X_{\dot{q}}, Y_{\dot{p}}, K_{\dot{v}}, M_{\dot{u}}$ non null.

\section{Drag}

Drag forces are generally expressed by a high order polynomial equation [8]. However, for the considered range of velocities $(0.5 \leq u \leq 2.5 \mathrm{~m} / \mathrm{s})$, the quadratic term is dominant. This assumption allows writing the drag forces and moments vector as

$$
\tau_{V}=-D_{V}\left(v_{r}\right) v_{r}
$$

where $D_{V}\left(v_{r}\right) \in \mathbb{R}^{6 \times 6}$.

As we will show later, drag coefficients depends on frontal, lateral and superior projected areas of the body of MARES. As in the case of added mass, symmetry related to the $\left\{x_{B}, z_{B}\right\}$ plane allows to simplify $D_{V}$.
For example, motion on the $y_{B}$-axis will originate a moment after $z_{B}$ (yaw moment). In other hand the same motion will not influence the linear motion after $x_{B}$ and $z_{B}$ or the angular motion after $y_{B}$ (pitch moment). Applying the same reasoning for the other coefficients, we obtain:

$$
\begin{aligned}
& D_{V}\left(v_{r}\right) \\
& =-\left[\begin{array}{cccccc}
X_{u|u|}|u| & 0 & 0 & 0 & X_{q|q|}|q| & 0 \\
0 & Y_{v|v|}|v| & 0 & Y_{p|p|}|p| & 0 & Y_{r|r|}|r| \\
0 & 0 & Z_{w|w|}|w| & 0 & Z_{q|q|}|q| & 0 \\
0 & K_{v|v|}|v| & 0 & K_{p|p|}|p| & 0 & 0 \\
M_{u|u|}|u| & 0 & M_{w|w|}|w| & 0 & M_{q|q|}|q| & 0 \\
0 & N_{v|v|}|v| & 0 & 0 & 0 & N_{r|r|}|r|
\end{array}\right],
\end{aligned}
$$

where $A_{i|i|}$ is the coefficient of the force induced after $A$ by $i$ velocity.

\section{Froude-Krylov forces}

This force is generated by the acceleration of the involving fluid in the presence of a body. It is expressed by

$$
\tau_{F K}\left(\dot{v}_{f}\right)=M_{F K} \dot{v}_{f} .
$$

MARES missions are often performed in a river or in ocean. Collected data from these missions shows that the variation of the fluid velocity is quasi-null, which imply $\dot{v}_{f} \approx 0 \Rightarrow \tau_{F K}\left(\dot{v}_{f}\right) \approx 0$.

\section{Restoring forces}

As stated before, these forces result from the weight and the buoyancy, given, in modulus by $W$ and $B$, respectively.

$$
\tau_{C}(\eta)=-\left[\begin{array}{c}
J_{1}^{-1}\left[\begin{array}{c}
0 \\
0 \\
W-B
\end{array}\right]---\frac{W}{--\underline{0}}-\left[\begin{array}{c}
0 \\
0 \\
W
\end{array}\right]-r_{C B} \times\left[\begin{array}{l}
0 \\
0 \\
B
\end{array}\right]
\end{array}\right]
$$

where $r_{C G}$ and $r_{C F}$ are positions of centers of gravity and buoyancy, respectively, related to the body-fixed referential. Assuming that the fixed-body referential coincides with the center of gravity and that $x_{C B}=y_{C B}=0$ we obtain

$$
\tau_{C}(\eta) \approx-\left[\begin{array}{c}
(W-B) \sin \theta \\
0 \\
(B-W) \cos \theta \\
0 \\
-z_{C F} B \sin \theta \\
0
\end{array}\right] .
$$

In the case of MARES, we consider that the angle after $x_{B}$ (roll) is $\varphi \approx 0$, translating its real behavior .

\section{Propulsion}

Linear force vectors of the propulsion (see fig. 4) are given by

$$
f_{P 1}=\left[\begin{array}{l}
1 \\
0 \\
0
\end{array}\right] F_{P 1} ; \quad f_{P 2}=\left[\begin{array}{l}
1 \\
0 \\
0
\end{array}\right] F_{P 2}
$$




$$
f_{P 3}=\left[\begin{array}{l}
0 \\
0 \\
1
\end{array}\right] F_{P 3} ; \quad f_{P 4}=\left[\begin{array}{l}
0 \\
0 \\
1
\end{array}\right] F_{P 4},
$$

where $F_{P i}$ is the force applied by the $i$-th thruster.

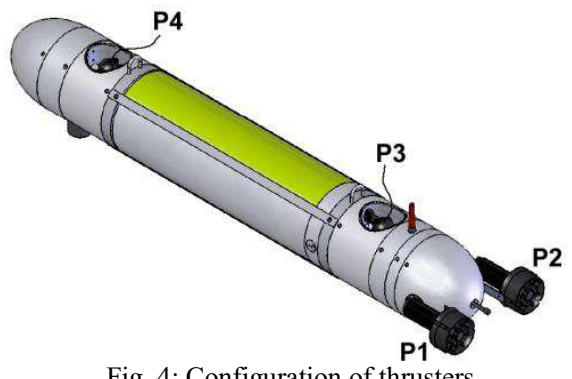

Fig. 4: Configuration of thrusters

The vector of forces and moments exercised by each thruster is given by

$$
\tau_{P i}=\left[\begin{array}{c}
f_{P i} \\
---- \\
r_{P i} \times f_{P i}
\end{array}\right] .
$$

For the total force and moment applied by the four thrusters, we obtain

$$
\tau_{P}=\sum_{i=1}^{4} \tau_{P i}
$$

Arranging this last expression, we may re-write it in the form

$$
\tau_{P}=P \cdot f_{p}
$$

where

$$
P=\left[\begin{array}{cccc}
1 & 1 & 0 & 0 \\
0 & 0 & 0 & 0 \\
0 & 0 & 1 & 1 \\
0 & 0 & 0 & 0 \\
z_{P 1} & z_{P 2} & -x_{P 3} & -x_{P 4} \\
-y_{P 1} & -y_{P 2} & 0 & 0
\end{array}\right] ; \quad f_{p}=\left[\begin{array}{c}
F_{P 1} \\
F_{P 2} \\
F_{P 3} \\
F_{P 4}
\end{array}\right]
$$

\section{Dynamic equation}

From subsections $\mathrm{B}$ and $\mathrm{C}$, the simplified dynamic equation results

$$
\tau_{\text {ext }}=\tau_{A}\left(\dot{v}_{r}, v_{r}\right)+\tau_{V}\left(v_{r}\right)+\tau_{C}(\eta)+\tau_{P}\left(v_{r}, n\right) .
$$

Substituting vectors by their expressions and manipulating algebraically, we obtain:

$$
\begin{aligned}
\left(M_{R B}+M_{A}\right) \dot{v}_{r}= & {\left[C_{R B}\left(v_{r}\right)+C_{A}\left(v_{r}\right)+D_{V}\left(v_{r}\right)\right] v_{r} } \\
& +\tau_{C}(\eta)+P \cdot f_{p} .
\end{aligned}
$$

\section{COEFFICIENTS DETERMINATION}

\section{A. Geometric model}

Coefficients that will be determined in following subsections depend on projected areas and volumes of different parts of MARES. Thus, the deducing of a geometric model will be useful.
MARES is formed by several geometric forms. The nose and the tail consist in two semi-ellipsoids, while the central part consists in a cylinder with two orifices (fig. 1-3). A cylindrical sonar hull is placed in the inferior part of the vehicle, as it may be seen in fig. 2-3. Vertical and horizontal thrusters are modeled as cylinders, both in motor hulls and in ducts. Considering this, we determine the radius of the vehicle as a function of $x_{B}$ in projections presented in fig. 1-3.

\section{Superior projection}

From fig. 1, it is possible to deduce the expression of the radius of the figure projected in the $\left\{x_{B}, y_{B}\right\}$ plane. Only the body hull is considered, neglecting thrusters, for the present. In this case, vertical orifices are also considered, subtracting their influence from the total radius along $x_{B}$. The expression of the hull radius in the superior as a function of $x_{B}$ results:

$R_{x y}\left(x_{B}\right)= \begin{cases}\frac{d}{2} \sqrt{1-\left(\frac{x_{B}-x_{e c}}{r_{e}}\right)^{2}} & ,\left[x_{c}, x_{e c}\right) \\ \frac{d}{2} & ,\left[x_{e c}, x_{f 1}-\frac{d_{f}}{2}\right) \\ \frac{d}{2}-\sqrt{\left(\frac{d_{f}}{2}\right)^{2}-\left(x_{B}-x_{f 1}\right)^{2}} & ,\left[x_{f 1}-\frac{d_{f}}{2}, x_{f 1}+\frac{d_{f}}{2}\right) \\ \frac{d}{2} & ,\left[x_{f 1}+\frac{d_{f}}{2}, x_{f 2}-\frac{d_{f}}{2}\right) \\ \frac{d}{2}-\sqrt{\left(\frac{d_{f}}{2}\right)^{2}-\left(x_{B}-x_{f 2}\right)^{2}} & ,\left[x_{f 2}-\frac{d_{f}}{2}, x_{f 2}+\frac{d_{f}}{2}\right) \\ \frac{d}{2} & ,\left[x_{f 2}+\frac{d_{f}}{2}, x_{e n}\right) \\ \frac{d}{2} \sqrt{1-\left(\frac{x_{B}-x_{e n}}{r_{e}}\right)^{2}} & ,\left[x_{e n}, x_{n}\right),\end{cases}$

where $d$ is the diameter of the hull, $d_{f}$ the diameter of orifices and $r_{e}=x_{e c}-x_{c}=x_{n}-x_{e n}$. For the intervals, report to fig. 1-2.

The radius of the contribution of the two horizontal propellers form is given by

$$
R_{P h x y}\left(x_{B}\right)=\left\{\begin{array}{lll}
d_{d} / 2 & , & {\left[x_{d}, x_{m i}\right)} \\
d_{m} / 2 & , & {\left[x_{m i}, x_{m f}\right) .}
\end{array}\right.
$$

Vertical thrusters influence is essentially due to the motor hull, neglecting duct and helices effects. Thus, it results in the following expressions for the radius of vertical thruster as a function of $x_{B}$ :

$R_{P v x y}\left(x_{B}\right)= \begin{cases}\sqrt{\left(\frac{d_{m}}{2}\right)^{2}-\left(x_{B}-x_{f 1}\right)^{2}},\left[x_{f 1}-\frac{d_{m}}{2}, x_{f 1}+\frac{d_{m}}{2}\right) \\ \sqrt{\left(\frac{d_{m}}{2}\right)^{2}-\left(x_{B}-x_{f 2}\right)^{2}},\left[x_{f 2}-\frac{d_{m}}{2}, x_{f 2}+\frac{d_{m}}{2}\right)\end{cases}$

\section{Lateral projection}

The lateral projection of the vehicle hull (fig. 2), i.e., in the $\left\{x_{B}, z_{B}\right\}$ allows determining of the radius defined as 


$$
R_{x Z}\left(x_{B}\right)= \begin{cases}\frac{d}{2} \sqrt{1-\left(\frac{x_{B}-x_{e c}}{r_{e}}\right)^{2}} & ,\left[x_{c}, x_{e c}\right) \\ \frac{d}{2} & ,\left[x_{e c}, x_{e n}\right) \\ \frac{d}{2} \sqrt{1-\left(\frac{x_{B}-x_{e n}}{r_{e}}\right)^{2}} & ,\left[x_{e n}, x_{n}\right)\end{cases}
$$

Notice that, in this case, we consider that the diameter of vertical orifices is sufficiently small in order to assume that respective concavities in lateral projection are negligible.

In this projection, part of propellers coincides with the vehicle hull, resulting in the following expression:

$$
R_{P x Z}\left(x_{B}\right)= \begin{cases}\frac{d_{d}}{2} & , x_{d} \leq x_{B}<x_{m i} \\ \frac{d_{m}}{2} & , x_{m i} \leq x_{B}<x_{c} .\end{cases}
$$

The sonar hull height is given by

$$
H_{S}\left(x_{B}\right)=z_{S f}-z_{S i} \quad, x_{S i} \leq x_{B}<x_{S f} .
$$

\section{Frontal projection}

The radius of the frontal projection of the hull (in the $\left\{y_{B}, z_{B}\right\}$ plane) along the $z_{B}$-axis is given by

$$
R_{y z}\left(z_{B}\right)=\sqrt{\left(\frac{d}{2}\right)^{2}-z_{B}^{2}} .
$$

The same projection of horizontal propellers results

$$
R_{P y z}\left(y_{B}\right)=\left\{\begin{array}{cc}
\sqrt{\left(\frac{d_{m}}{2}\right)^{2}-\left(y_{B}+y_{m}\right)^{2}} & {\left[-y_{m}-\frac{d_{m}}{2},-y_{m}+\frac{d_{m}}{2}\right)} \\
\sqrt{\left(\frac{d_{m}}{2}\right)^{2}-\left(y_{B}-y_{m}\right)^{2}} & {\left[y_{m}-\frac{d_{m}}{2}, y_{m}+\frac{d_{m}}{2}\right) .}
\end{array}\right.
$$

The length of the projected frontal area of the sonar hull is given by

$$
L_{S}\left(z_{B}\right)=2 y_{S} \quad, \quad z_{S i} \leq z_{B} \leq z_{S f} .
$$

\section{B. Rigid body coefficients}

The vehicle mass is $m=32 \mathrm{~kg}$, essentially concentrated on the central tight part of MARES, in the interval $\left[x_{c i}, x_{c f}\right]$, and on thrusters, whose densities are different. Remaining parts of the hull are hollow and filled by involving water when submerged. Thus, neglecting the weight of the hull, we assume that there are three homogeneous density zones, presented in table I.

TABLE I

DENSITIES OF MARES

\begin{tabular}{|c|c|l|}
\hline Density & Value $\left[\mathrm{kg} / \mathrm{m}^{3}\right]$ & \multicolumn{1}{c|}{ Description } \\
\hline$\rho_{\text {est }}$ & $1.36 \cdot 10^{3}$ & Tight volume density \\
\hline$\rho_{\text {perm }}$ & $1.03 \cdot 10^{3}$ & Permeable volume density (water density) \\
\hline$\rho_{p}$ & $3.84 \cdot 10^{3}$ & Thruster volume density \\
\hline
\end{tabular}

Defining $\rho\left(x_{B}\right)$ as the density of the MARES AUV as a function of $x_{B}$, we may compute moments of inertia defined in section III as presented below:

$$
\begin{gathered}
I_{x x}=\int_{V} \rho_{B}\left(y_{B}^{2}+z_{B}^{2}\right) d V ; \\
I_{y y}=\int_{V} \rho_{B}\left(x_{B}^{2}+z_{B}^{2}\right) d V ; \\
I_{z z}=\int_{V} \rho_{B}\left(x_{B}^{2}+y_{B}^{2}\right) d V .
\end{gathered}
$$

Using the geometric model, it results

$$
\begin{aligned}
I_{x x}=\int_{-d / 2}^{d / 2} \int_{0}^{2 \pi} \int_{0}^{R_{y z}\left(z_{B}\right)} & \rho_{t}\left(r^{2} \cos ^{2} \varphi+r^{2} \sin ^{2} \varphi\right) r d r d \varphi d z_{B} \\
& +2 \int_{x_{m i}}^{x_{m f}} \int_{d / 2}^{d / 2+d_{m}} \rho_{p}\left(y^{2}\right. \\
& \left.+2\left(R_{P y z}\left(y_{B}\right)\right)^{2}\right) d y_{B} d x_{B} \\
I_{y y} \quad=\int_{x_{c}}^{x_{n}} \int_{0}^{2 \pi} \int_{0}^{R_{x z}\left(x_{B}\right)} & \rho\left(x_{B}\right)\left(x_{B}^{2}+r^{2} \sin ^{2} \varphi\right) r d r d \varphi d x_{B} \\
& +2 \int_{x_{m i}}^{x_{m f}} \int_{0}^{2 \pi} \int_{0}^{R_{P x z}\left(x_{B}\right)} \rho_{p}\left(x_{B}^{2}\right. \\
& \left.+r^{2} \sin ^{2} \varphi\right) r d r d \varphi d x_{B} \\
& +\int_{x_{c}}^{x_{n}} \int_{L_{m}}^{2 \int_{P v}\left(x_{B}\right)} \rho_{0}\left(x_{B}^{2}\right. \\
& \left.+r^{2} \sin ^{2} \varphi\right) r d r d \varphi d x_{B} \\
I_{z z} \approx I_{y y}, &
\end{aligned}
$$

where $\rho_{t}=\frac{1}{L} \int_{L} \rho\left(x_{B}\right) d x_{B}$ is the mean density of MARES and $L_{m}$ motors hull length.

TABLE II

MOMENT OF INERTIA VALUES

\begin{tabular}{|c|c|}
\hline Moment of inertia & Value $\left[\mathrm{kg} \cdot \mathrm{m}^{2}\right]$ \\
\hline$I_{x x}$ & $1.55 \cdot 10^{-1}$ \\
\hline$I_{y y}$ & $4.73 \cdot 10^{0}$ \\
\hline$I_{z z}$ & $4.73 \cdot 10^{0}$ \\
\hline
\end{tabular}

\section{Added mass coefficients}

The axial term $X_{\dot{u}}$ is determined using the equation (32) from $[8, \mathrm{p} .41]$. We need to approximate the vehicle by an ellipsoid with a minor axis $a=l / 2$ and a major axis $b=d / 2$.

$$
X_{\dot{u}}=-\frac{\alpha_{0}}{2-\alpha_{0}} m
$$

where $m$ is the total vehicle mass, and $\alpha_{0}$ is given by [8]

$$
\alpha_{0}=\frac{2\left(1-e^{2}\right)}{e^{3}}\left[\frac{1}{2} \ln \left(\frac{1+e}{1-e}\right)-e\right]
$$

and

$$
e=1-\left(\frac{b}{a}\right)^{2}
$$

Based on [3] and [10], we assume that the major contribute to the added mass are the vehicle hull, the horizontal propellers and the sonar transducer. Fluid masses displaced by 
the AUV, per unit of a transversal "slice" of these three contributions, are respectively:

$$
\begin{aligned}
& m_{a}\left(x_{B}\right)=\rho_{f} \int_{0}^{2 \pi} \int_{0}^{R x y\left(x_{B}\right)} r d r d \varphi=\rho_{f} \pi R\left(x_{B}\right)^{2} \\
& m_{a P}\left(x_{B}\right)=\rho_{f} \int_{0}^{2 \pi} \int_{0}^{R_{P h x y}\left(x_{B}\right)} r d r d \varphi=\rho_{f} \pi R_{P}\left(x_{B}\right)^{2} \\
& m_{a S}\left(x_{B}\right)=2 \rho_{f} \int_{0}^{x_{B}} H_{S} \sqrt{y_{S}^{2}-\left(x-x_{c S}\right)^{2}} d x
\end{aligned}
$$

where $R\left(x_{B}\right)$ is the hull radius, $R_{P}\left(x_{B}\right)$ is the propeller radius, $H_{S}$ is the sonar hull height and $x_{C S}$ the sonar hull base center.

The motions in $y_{B}$ e $z_{B}$ directions cause different forces and moments due to the existence of verticals holes in the hull. In $y_{B}$ or yaw motion, the fluid present in the holes is transported with the vehicle, but in $z_{B}$ or pitch motion, the fluid does not suffer any acceleration. This last case implies that the fluid passes through the holes without exercising any force or moment on the vehicle.

We approach the holes by cylinders with a diameter $d_{f}$ and constant height $h_{f}$. We can now define the added mass per unit length, due to holes:

$$
m_{a f}\left(x_{B}\right)= \begin{cases}\rho_{f} h_{f} \sqrt{\left(\frac{d_{f}}{2}\right)^{2}-\left(x_{B}-x_{f 1}\right)^{2}}, & x_{f 1}-\frac{d_{f}}{2} \leq x_{B} \\ \rho_{f} h_{f} \sqrt{\left(\frac{d_{f}}{2}\right)^{2}-\left(x_{B}-x_{f 2}\right)^{2}}, & <x_{f 1}+\frac{d_{f}}{2} \\ & x_{f 2}-\frac{d_{f}}{2} \leq x_{B} \\ 0, & <x_{f 2}+\frac{d_{f}}{2} \\ \text { Other }\end{cases}
$$

In expression (41), we present expressions to determine some added mass terms. The other terms can be determined by analogy.

$$
\begin{gathered}
Z_{\dot{w}}=-\left[\int_{x_{C}}^{x_{n}} m_{a}\left(x_{B}\right) d x_{B}+2 \int_{x_{d}}^{x_{m f}} m_{a P}\left(x_{B}\right) d x_{B}\right. \\
\left.+\int_{x_{S i}}^{x_{S f}} m_{a S}\left(x_{B}\right) d x_{B}-\int_{x_{c}}^{x_{n}} m_{a f}\left(x_{B}\right) d x_{B}\right] \\
N_{\dot{r}}=-\left[\int_{x_{C}}^{x_{n}} x_{B}^{2} m_{a}\left(x_{B}\right) d x_{B}+2 \int_{x_{d}}^{x_{m f}} x_{B}^{2} m_{a P}\left(x_{B}\right) d x_{B}\right. \\
\left.+\int_{x_{S i}}^{x_{S f}} x_{B}^{2} m_{a S}\left(x_{B}\right) d x_{B}\right] \\
Y_{\dot{r}}=\left[\int_{x_{C}}^{x_{n}} x_{B} m_{a}\left(x_{B}\right) d x_{B}+2 \int_{x_{d}}^{x_{m f}} x_{B} m_{a P}\left(x_{B}\right) d x_{B}\right. \\
\left.+\int_{x_{S i}}^{x_{S f}} x_{B} m_{a S}\left(x_{B}\right) d x_{B}\right]
\end{gathered}
$$

for the limits of integration, see fig. 2 and fig. 3 .

The most important added mass coefficients are shown in table III.

TABLE III

ADDED MASS COEFFICIENT VALUES

\begin{tabular}{|c|c|}
\hline Coefficient & Value \\
\hline$X_{\dot{u}}$ & $-1.74 \cdot 10^{0}$ \\
\hline$Y_{\dot{v}}$ & $-4.28 \cdot 10^{1}$ \\
\hline$Z_{\dot{w}}$ & $-3.88 \cdot 10^{1}$ \\
\hline$K_{\dot{p}}$ & $-2.05 \cdot 10^{-1}$ \\
\hline$M_{\dot{q}}$ & $-5.59 \cdot 10^{0}$ \\
\hline
\end{tabular}

\begin{tabular}{|c|c|}
\hline Coefficient & Value \\
\hline$N_{\dot{r}}$ & $-6.32 \cdot 10^{0}$ \\
\hline$Y_{\dot{r}}$ & $2.89 \cdot 10^{-2}$ \\
\hline$Z_{\dot{q}}$ & $-2.10 \cdot 10^{-1}$ \\
\hline$M_{\dot{\mathrm{w}}}$ & $-2.10 \cdot 10^{-1}$ \\
\hline$N_{\dot{v}}$ & $2.89 \cdot 10^{-2}$ \\
\hline
\end{tabular}

\section{Drag}

In this work, we consider only quadratic drag terms. We assume that the linear and angular speeds are sufficiently high to neglect linear terms. The terms greater than second order will be also neglect, assuming that their effects are small comparing to quadratic terms.

From [8] and [11] we have the axial force in the $x_{B}$ direction expressed as:

$$
f_{d u}\left(u_{r}\right)=-\frac{1}{2} \rho_{f} C_{D e} A_{y z} u_{r}\left|u_{r}\right|
$$

where $\rho_{f}=1.026 \cdot 10^{3} \mathrm{~kg} / \mathrm{m}^{3}$ is the fluid density, $C_{D e}$ is the drag coefficient of an ellipsoidal body depending on the hull form and $A_{y z}$ is the vehicle projected area in the plane formed by $y_{B}$ and $z_{B}$ axes.

The determination of drag coefficients can result on wrong estimations [3] due to the theory behind it witch is often based on empirical expressions or experimental results. The best way to estimate them is by experimental tests. However, these estimates are useful as first approach to the characterization of the vehicle motion.

The drag coefficient $C_{D e}$ depends on Reynolds Number $R_{e}$, given by:

$$
R_{e}=\frac{U l}{v}
$$

where $U$ is the axial speed in the $x_{B}$-axis, $l$ the vehicle length and $v$ the fluid viscosity. We assume $U=1 \mathrm{~m} / \mathrm{s}$ which is a typical value for the vehicle velocity, $l=1.5 \mathrm{~m}$ and $v=$ $1.005 \cdot 10^{-6} \mathrm{~m}^{2} / \mathrm{s}$ at $T=20^{\circ} \mathrm{C}$. These values give $R_{e}=$ 1.5e6. This implies that the vehicle motion is between the laminar and the turbulent flow [11].

Using laminar theory, we have the following drag coefficient:

$$
C_{\text {Dle }}=0.44 \frac{d}{l}+4 C_{f} \frac{l}{d}+4 C_{f}\left(\frac{d}{l}\right)^{\frac{1}{2}},
$$

where $d$ is the vehicle diameter, and $C_{f}$ is given by [9]:

$$
C_{f}=\frac{0.075}{\left(\log _{10} R_{e}-2\right)^{2}}
$$

Resulting on $C_{D l e}=0.2$.

The turbulence theory gives $C_{D t e}=0.08$. The experimental results obtained by [3] to determine the drag coefficient point to a value close to $C_{D l e}$. Taking into account that the dimensions and the speed of operation are similar, we consider:

$$
C_{D}=C_{D l e}
$$

We are now in condition to determine the axial term in $x_{B}$ direction: 


$$
\begin{aligned}
X_{u|u|}=-\frac{1}{2} \rho_{f}\left[2 C_{D e}\right. & \int_{-\frac{d}{2}}^{\frac{d}{2}} R_{y z}\left(z_{B}\right) d z_{B} \\
& +2 C_{D e} \int_{\frac{d}{2}}^{y_{m}+\frac{d_{m}}{2}} R_{P y z}\left(y_{B}\right) d y_{B} \\
& +2 C_{D e} \int_{-\frac{d}{2}}^{-y_{m}-\frac{d_{m}}{2}} R_{P y z}\left(y_{B}\right) d y_{B} \\
& \left.+C_{D c S} \int_{z_{S i}}^{z_{S f}} L_{S y z}\left(z_{B}\right) d z_{B}\right] .
\end{aligned}
$$

For the limits of integration, see fig.2 and fig.3.

The motion in the $y_{B}$ and $z_{B}$ leads a flow around the vehicle that we approximate by a cylinder. According to [11] and [12], for a cylinder with a ratio $l / d \approx 7.5$, the drag coefficient is:

$$
C_{D c}=0.8
$$

The sonar hull under the vehicle is also cylindrical with a ratio $l_{S} / d_{S} \approx 1$. From [11] and [12], we obtain a drag coefficient equal to:

$$
C_{D c S}=0.68
$$

The drag coefficient terms are determined as shown below:

$$
\begin{gathered}
Y_{v|v|}=-\frac{1}{2} \rho_{f}\left[2 C_{D c} \int_{x_{c}}^{x_{n}} R_{x z}\left(z_{B}\right) d x_{B}+2 C_{D c} \int_{x_{d}}^{x_{c}} R_{P x z}\left(x_{B}\right) d x_{B}\right. \\
\left.+C_{D c S} \int_{x_{S i}}^{x_{S f}} H_{S x z}\left(x_{B}\right) d x_{B}\right] \\
M_{q|q|}=-\frac{1}{2} \rho_{f}\left[2 C_{D c} \int_{x_{c}}^{x_{n}}\left|x_{B}\right|^{3} R_{x y}\left(x_{B}\right) d x_{B}\right. \\
\left.+2 C_{D c} \int_{x_{d}}^{x_{m f}} 2\left|x_{B}\right|^{3} R_{P x y}\left(x_{B}\right) d x_{B}\right] \\
Z_{q|q|}=\frac{1}{2} \rho_{f}\left[2 C_{D c} \int_{x_{c}}^{x_{n}} x_{B}\left|x_{B}\right| R_{x y}\left(x_{B}\right) d x_{B}\right. \\
\left.+2 C_{D c} \int_{x_{d}}^{x_{m f}} 2 x_{B}\left|x_{B}\right| R_{P x y}\left(x_{B}\right) d x_{B}\right] \\
M_{w|w|}=\frac{1}{2} \rho_{f}\left[2 C_{D c} \int_{x_{c}}^{x_{n}} x_{B} R_{x y}\left(x_{B}\right) d x_{B}\right. \\
\left.+2 C_{D c} \int_{x_{d}}^{x_{m f}} 2 x_{B} R_{P x y}\left(x_{B}\right) d x_{B}\right]
\end{gathered}
$$

for the limits of integration, see fig. 2 and fig. 3

The remaining drag terms can be determined by analogy. The most important drag coefficients are shown in table III.

TABLE III

DRAG COEFFICIENT VALUES

\begin{tabular}{|c|c|}
\hline Coefficient & Value \\
\hline$X_{u|u|}$ & $-4.05 \cdot 10^{0}$ \\
\hline$Y_{v|v|}$ & $-1.13 \cdot 10^{2}$ \\
\hline$Z_{w|w|}$ & $-1.15 \cdot 10^{2}$ \\
\hline$K_{p|p|}$ & $-2.29 \cdot 10^{-2}$ \\
\hline$M_{q|q|}$ & $-4.30 \cdot 10^{0}$ \\
\hline
\end{tabular}

\begin{tabular}{|c|c|}
\hline Coefficient & Value \\
\hline$N_{r|r|}$ & $-1.57 \cdot 10^{0}$ \\
\hline$Y_{r|r|}$ & $1.88 \cdot 10^{0}$ \\
\hline$Z_{q|q|}$ & $-5.95 \cdot 10^{0}$ \\
\hline$M_{w|w|}$ & $-8.25 \cdot 10^{0}$ \\
\hline$N_{v|v|}$ & $2.38 \cdot 10^{0}$ \\
\hline
\end{tabular}

\section{LIMITS ON MOTION}

In this section, we intend to identify limits in some maneuvers. This exercise will allow a better knowledge of the behavior of MARES due to actuation limits [13]. We will start presenting simple motions maneuvers and continue with composed motions.

We assume that the maximum actuation force that it can be performed by propellers of MARES is $F_{p \max }=21.5 \mathrm{~N}$.

\section{A. Longitudinal motion}

Considering that the vehicle moves only after $x_{B}$ at constant speed $u$, we get

$$
v=\left[\begin{array}{llllll}
u & 0 & 0 & 0 & 0 & 0
\end{array}\right]^{T},
$$

which imply that its time derivative is $\dot{v}=0_{6 \times 6}$.

Extracting the first line of (24), we obtain

$$
X_{u|u|} u|u|-(W-B) \sin \theta+F_{P 1}+F_{P 2}=0 .
$$

Assuming that the pitch angle $\theta=0$, we easily obtain the maximum forward velocity as presented below:

$$
\begin{aligned}
u_{\max } & =\sqrt{\frac{2 F_{p \max }}{-X_{u|u|}}} \\
& =3.2 \mathrm{~m} / \mathrm{s} .
\end{aligned}
$$

\section{B. Diving motion}

For this motion, we assume that the vehicle dive at constant velocity $w\left(\operatorname{after} z_{B}\right)$ with null pitch $(\theta=0)$. Remaining linear and angular velocities are considered to be zero. Using the third line of the equation (24), we get

$$
-Z_{w|w|} w|w|=F_{P 3}+F_{P 4}
$$

From this expression, we determine the maximum diving velocity for $\theta=0$ as

$$
\begin{aligned}
w_{\text {max }} & =\sqrt{\frac{2 F_{\text {max }}}{-Z_{w|w|}}} \\
& =6.09 \cdot 10^{-1} \mathrm{~m} / \mathrm{s} .
\end{aligned}
$$

\section{Rotational motion}

As in previous subsections, only a velocity is considered. In this case we assume that $r$ (velocity after $z_{B}$ ) is a positive constant while the remaining linear and angular velocities are nulls. Extracting and manipulating the sixth line of the motion dynamic equation (24), we obtain, after simplification

$$
N_{r|r|} r|r|=y_{P 1} F_{P 1}+y_{P 2} F_{P 2}
$$

Knowing that $y_{P 2}=-y_{P 1}$, the maximum yaw velocity is determined as shown:

$$
\begin{aligned}
r_{\text {max }} & =\sqrt{\frac{2 y_{P 1} F_{p \max }}{N_{r|r|}}} \\
& =6.09 \cdot 10^{-1} \mathrm{rad} / \mathrm{s} .
\end{aligned}
$$

\section{Circumference maneuver}

In this analysis, we consider that velocities $u$ and $r$ are positive constants in steady state. This fact could imply non null lateral velocity $v\left(\right.$ after $\left.y_{B}\right)$. Thus,

$$
v=\left[\begin{array}{llllll}
u & v & 0 & 0 & 0 & r
\end{array}\right] .
$$


This maneuver is illustrated in fig. 5. We define $R$ as the curvature radius of the described circumference. In steady state, we may conclude that

$$
r=\frac{\sqrt{u^{2}+v^{2}}}{R}
$$

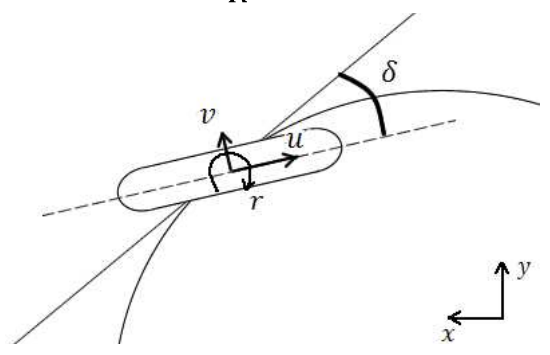

Fig. 5: Motion with $u \neq 0$ e $r \neq 0$.

From the second line of the dynamic equation, it is possible to deduce

$$
|v| v=-\frac{\left(X_{\dot{u}} u-m u+Y_{|r| r}|r|\right) r}{Y_{|v| v}}
$$

whose resolution for $u, v, r>0$ is impossible. This implies that the nose points to the center of the circumference.

In other hand, selecting the first and the sixth lines, we determine the following expressions that may be seen as common and differential mode expressions, respectively:

$$
\begin{gathered}
F_{p 1}+F_{p 2}=\left(Y_{\dot{v}} v+Y_{\dot{r}} r\right) r-m r v-X_{|u| u}|u| u, \\
F_{p 1}-F_{p 2} \\
=\frac{\left(-Y_{\dot{v}} v-Y_{\dot{r}} r\right) u+X_{\dot{u}} u v-N_{|v| v}|v| v-N_{|r| r}|r| r}{y_{p}} .
\end{gathered}
$$

Adding and subtracting (61) to (60), we obtain, respectively:

$$
\begin{aligned}
& F_{p 1} \\
& =\frac{1}{2}\left[\left(Y_{\dot{v}} v+Y_{\dot{r}} r\right) r-m r v-X_{|u| u}|u| u\right. \\
& \left.+\frac{\left(-Y_{\dot{v}} v-Y_{\dot{r}} r\right) u+X_{\dot{u}} u v-N_{|v| v}|v| v-N_{|r| r}|r| r}{y_{p}}\right]
\end{aligned}
$$

and

$$
\begin{aligned}
& F_{p 2} \\
& =\frac{1}{2}\left[\left(Y_{\dot{v}} v+Y_{\dot{r}} r\right) r-m r v-X_{|u| u}|u| u\right. \\
& \left.-\frac{\left(-Y_{\dot{v}} v-Y_{\dot{r}} r\right) u+X_{\dot{u}} u v-N_{|v| v}|v| v-N_{|r| r}|r| r}{y_{p}}\right] .
\end{aligned}
$$

Solving numerically the equation system composed by (59), (60), (63) and (64), for $u=1 \mathrm{~m} / \mathrm{s}$, we obtain the following graph of force applied by horizontal thrusters.

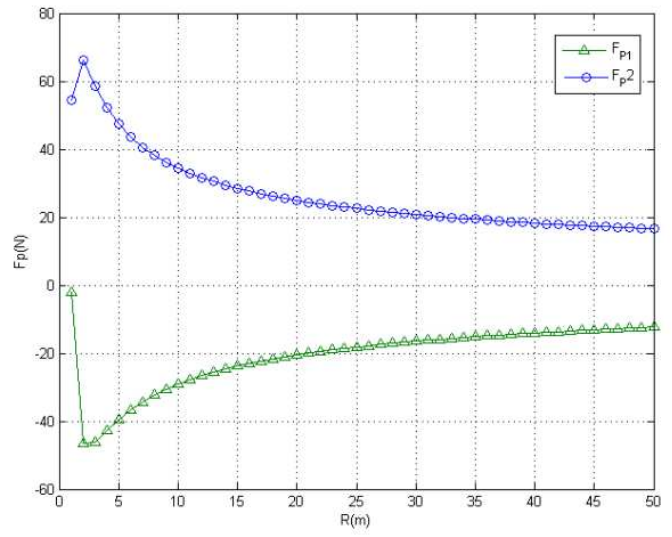

Fig. 6: Forces applied by horizontal thrusters as functions of curvature

This result is paradoxical, given that, as we can conclude from the last figure, the moment performed by horizontal propellers is opposed to the rotation. Exercised moment is negative after the $z_{B}$-axis, while the rotation is positive. This fact is due to the Munk moment expressed by the term given by $Y_{\dot{v}} u v$. According to [14] and [15], this moment tends to turn the vehicle (streamlined body) perpendicular to its motion. It is explained by the non homogeneous pressure on the hull of MARES. In this case, the nose area would have greater pressure than the tail.

Experimentally, it is difficult to observe this phenomenon and, for the considered range of velocities, it is compensated by drag terms that we have neglected, as $N_{u v}$, whose value is difficult to predict. According to [15], the motion at a considerable angle of attack generates a boundary layer near from the body hull, which causes an additional drag and moment opposed to Munk moment. In order to approach the model to experimental data, we decide to neglect Munk moment terms as $Y_{\dot{v}} u v, X_{\dot{u}} u v, Z_{\dot{w}} u w$ and $X_{\dot{u}} u w$.

With these assumptions, for the motion with $u, r>0$, thruster P1 will be the first that reach the saturation value $F_{\text {pmax }}$, as expected. Solving the system of equations given by (59), (60) and (63) for $F_{P 1}=F_{p \max }$, it is possible to determine the maximum forward velocity $u_{\max }$ as a function of curvature radius, $R$. The result is presented in the following figure.

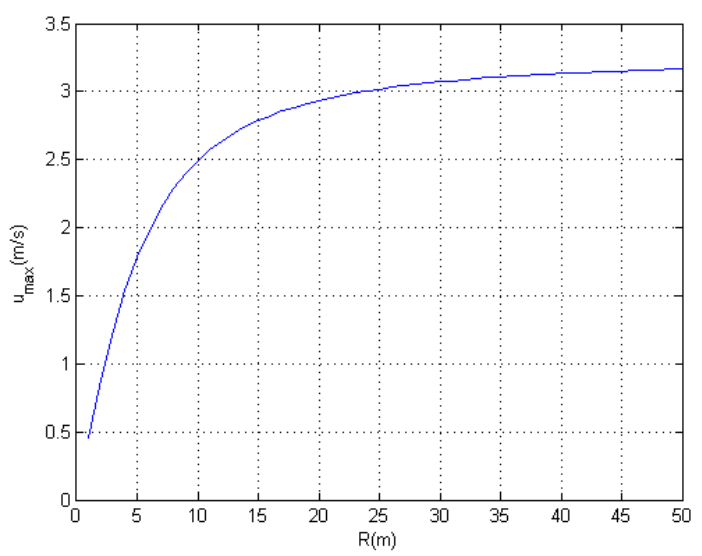

Fig. 7: Maximum forward velocity as a function of curvature 
Another interesting result that could be extracted is the angle formed between the vehicle and the circumference tangent, $\delta$ (see fig. 5). In fig. 8 , we present its value as a function of curvature radius.

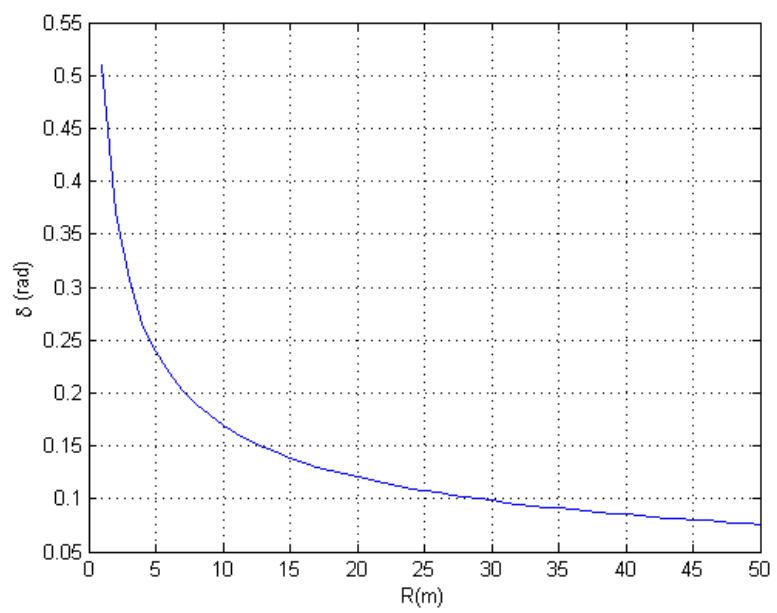

Fig. 8: Angle between vehicle and tangent of the circumference as a function of curvature

\section{EXPERIMENTAL RESULTS}

In order to validate and correct the model of MARES, experimental tests were performed. They were carried out in the river of Douro, near from Porto. During maneuvers, controllers supply voltage references to thrusters and localization data is collected.

\section{A. Longitudinal drag}

A horizontal velocity controller [5] was set to a linear motion at constant forward velocity $u_{r e f}=1 \mathrm{~m} / \mathrm{s}$ and $\theta=0$. The flow effect is neglected, taking into account that its velocity is smaller compared to the vehicle one. The filtered data of the absolute position derivative, in order of time, is presented in fig. 9. The noise is due to the acoustic localization error, whose effect is considerable in time derivatives. In steady state, the average of the velocity is $u \approx 0.95 \mathrm{~m} / \mathrm{s}$.

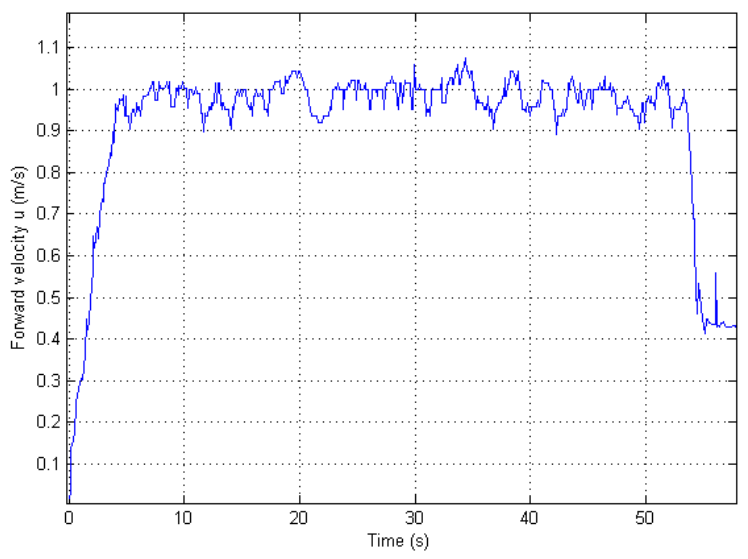

Fig. 9: Experimental forward velocity $u$

For this velocity, we estimate that the force of horizontal thrusters is $F_{P 1} \approx F_{P 2} \approx 4.7 N$. This estimation is obtained from experimental tests performed with MARES thrusters. Using (51), we deduce

$$
\begin{aligned}
X_{u|u|} & =-\frac{F_{P 1}+F_{P 2}}{u|u|} \\
& \approx-10 \mathrm{~kg} \cdot \mathrm{m}^{-1}
\end{aligned}
$$

This implies a maximum forward velocity $u_{\max } \approx 2 \mathrm{~m} / \mathrm{s}$ for the corrected model, which is close to the observed real behavior.

\section{B. Diving drag}

As in the previous subsection, a vertical velocity controller was set in order to dive at constant velocity $w_{\text {ref }}=0.4 \mathrm{~m} / \mathrm{s}$. The system response is shown in the next figure.

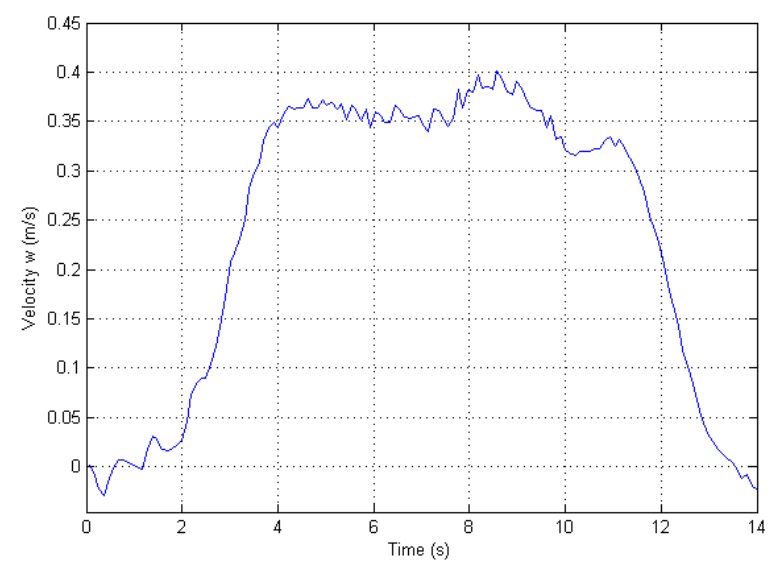

Fig. 10: Experimental transversal velocity $w$

We assume that the velocity is constant in the interval $t \in[4,7](s)$ and equal to $w=0.36 \mathrm{~m} / \mathrm{s}$. The collection of data related to the voltage applied to vertical thrusters allows estimating the force applied by each one: $F_{P 3} \approx F_{P 4} \approx 7.5 \mathrm{~N}$. Therefore, using (53), it is possible to determine the drag coefficient, as demonstrated below:

$$
\begin{aligned}
Z_{w|w|} & =-\frac{F_{P 3}+F_{P 4}}{w|w|} \\
& \approx 1.22 \cdot 10^{2} \mathrm{~kg} \cdot \mathrm{m}^{-1}
\end{aligned}
$$

This result is very close to the theoretical one.

\section{Rotational drag}

Applying only a deferential mode to horizontal thruster such as $F_{P 1} \approx-F_{P 2} \approx 15 N$ and collecting the data related to yaw angle, it is possible to determine the rotational drag coefficient $N_{r|r|}$. The response of the vehicle is shown in the fig. 11 . 


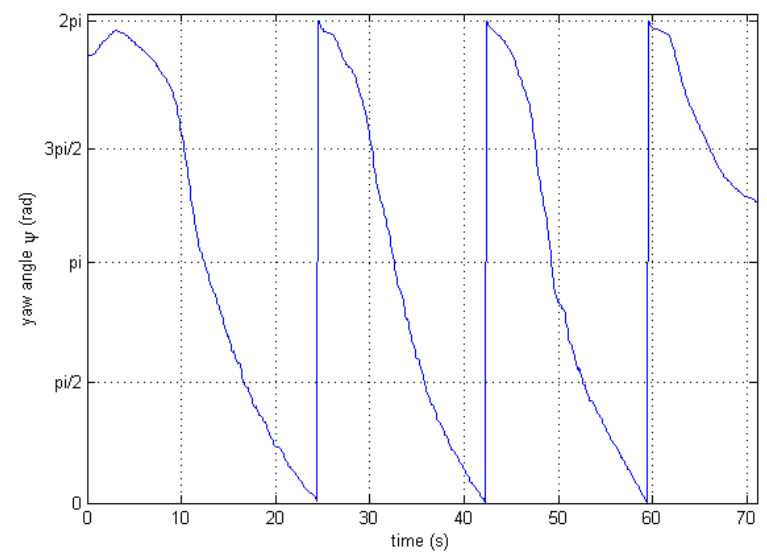

Fig. 11: Experimental yaw angle $\psi$

It was expected that the variation of the yaw angle would be linear but the presence of possible magnetic fields in the interior of the hull affects the collected data.

However, the angular velocity can be easily extracted, considering the period of the wave form. In steady state, it results $r=3,59 \cdot 10^{-1} \mathrm{rad} / \mathrm{s}$. Using (56), we easily deduce

$$
N_{r|r|}=\frac{y_{P 1} F_{P 1}+y_{P 2} F_{P 2}}{r|r|},
$$

from which we determine that $N_{r|r|}=2.51 \cdot 10^{1} \mathrm{~kg} \cdot \mathrm{m}^{2}$.

Though experiments show a value in the same range as the determined theoretically, this result may be uncertain given that we can not guarantee that forces applied by thrusters are the same as previously defined. The reduced distance between horizontal actuators may generate disturbances in each other, due to the fluid motion, mainly when exercised forces are opposite.

\section{CONCLUSIONS}

This paper proposes a modeling method of an autonomous underwater vehicle. First, we have presented the main characteristics of MARES, a small-sized AUV developed at the University of Porto, whose motion can be performed only in the vertical plane through its two vertical thrusters.
Next, fundamental concepts and theory were exposed as the so-used in robotic kinematic and the rigid body dynamic from the Newton's second law. Main forces and moments for underwater vehicles were identified and a general modeling method was stated, distinguishing every kind of actuation on the body. Through geometric characteristics of MARES, it was possible to deduce a simplified tridimensional model and determine its coefficients whose deducing effort was done in order to obtain an accuracy translating of the real dynamic, this is why every technical features were considered.

We have followed with a theoretical analysis of several simple and composed motions, where we have identified and corrected some terms in order to obtain a better approach to the real behavior.

Finally, some experimental results were presented and, from steady state analysis, it was possible to compute estimated main drag coefficients though there are some uncertainties in the estimation of thruster actuation forces.

\section{REFERENCES}

[1] A. Matos and N. Cruz, Underwater Vehicles, Chapter 17. Austria: InTech, 2009.

[2] N. Cruz and A. Matos, "The MARES AUV, a Modular Autonomous Robot for Environment Sampling". Québec, Canada : Proceedings of the MTS-IEEE Conference Oceans'2008, 2008.

[3] T. Prestero, Verification of a Six-Degree of Freedom Simulation Model for the REMUS Autonomous Underwater Vehicle, MSc Thesis. MIT, Massachussets, 2001.

[4] A. P. Aguiar and J. P. Hespanha, "Trajectory-Tracking and PathFollowing of Underactuated Autonomous Vehicles with Parametric Modeling Uncertainty". IEEE transaction on automatic control, Vol. 52, no. 8, pp. 1362-1379, 2007.

[5] B. Ferreira, M. Pinto, A. Matos and N. Cruz, "Control of the MARES autonomous underwater vehicle". Biloxi, USA: Proceedings of the MTS-IEEE Conference Oceans'09, 2009.

[6] R. Siegwart and I. R. Nourbakhsh, Introduction to Autonomous Mobile Robots. Cambridge, USA: MIT Press, 2004.

[7] T.I. Fossen, Nonlinear modelling and control of underwater vehicle. Trodheim, Norway: PhD thesis, 1991.

[8] T. I. Fossen, Guidance and control of ocean vehicles. Chichester, England : John Wiley and Sons, 1994.

[9] O. M. Faltinsen, Hydrodynamic of high speed marine vehicles. USA : Cambridge, 2005.

[10] D. Yue, Marine Hydrodinamics: lecture 14. Massachusetts, USA: MIT, 2005.

[11] S. F. Hoerner, Fluid-dynamic drag. USA : edited by author, 1965.

[12] F. M. White, Fluid mechanics. Boston, USA: McGraw-Hill, 1999.

[13] B. Ferreira, Modelação e controlo de veículo submarino com quatro graus de liberdade. Porto, Portugal: MSc thesis edited by author, 2009.

[14] M. Triantafyllou and S. Franz, Maneuvering and Control of Marine Vehicles. Cambridge, Massachusetts, USA: edited by authors, 2003.

[15] A.Tyagi, D. Sen, "Calculation of transverse hydrodynamic coefficients using computational fluid dynamic approach". Ocean Engineering, Volume 33, Issues 5-6, April 2006, Pages 798-809. 